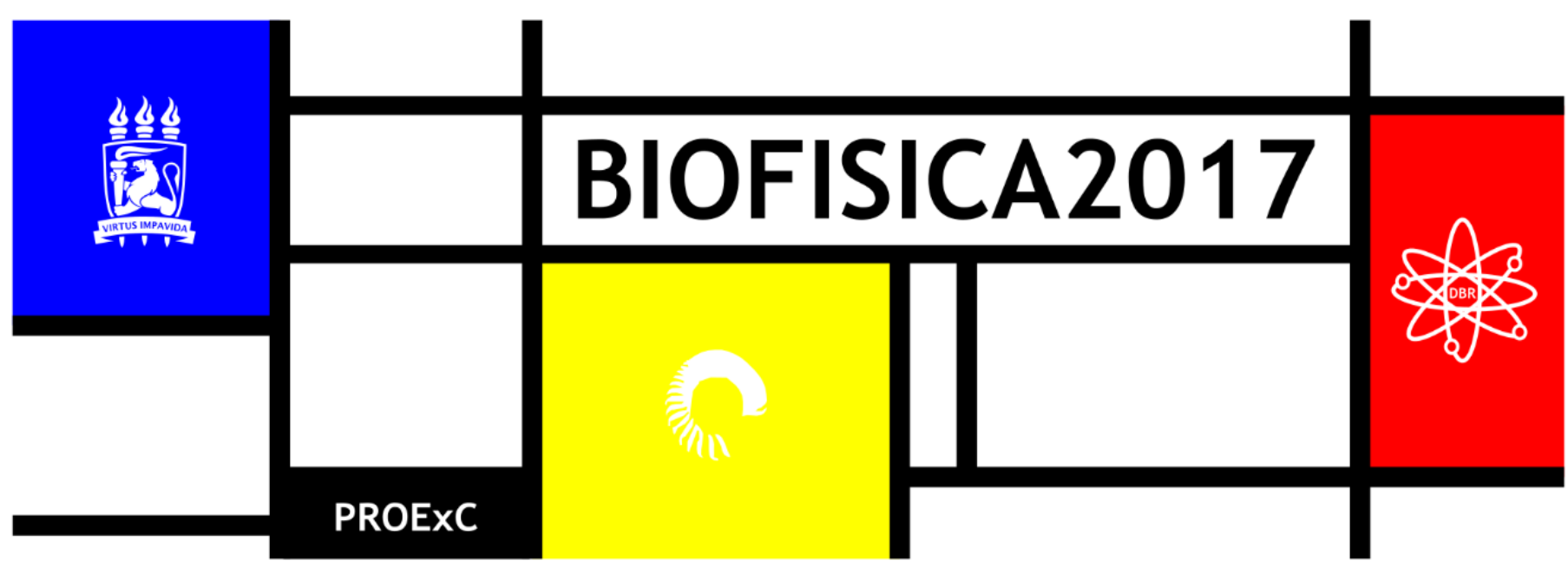

\title{
BIOACUMULAÇÃO DE ELEMENTOS TRAÇOS EM PLANTAS DA MATA ATLÂNTICA
}

\author{
Paulo Correia da S. Neto*1, Luiz J. Da Silva Neto ${ }^{1}$, Elvis J. De França ${ }^{1}$ \\ ${ }^{1}$ Centro Regional de Ciências Nucleares do Nordeste \\ *paulo.cdsn@gmail.com
}

\section{INTRODUÇÃO}

Os elementos químicos traços ocorrem naturalmente no ambiente. Alguns são essenciais, em baixas concentrações, para manter o metabolismo adequado dos seres vivos (FAIRBROTHER et al, 2007; $\mathrm{XU}$ et al., 2006), porém tóxicos em níveis mais elevados. Alguns elementos químicos não possuem funções biológicas bem definidas (SCHÜÜRMANN, 1998), além de serem altamente persistentes e não biodegradáveis com meias-vidas biológicas longas. Sua acumulação em solos, e na vegetação, em níveis ambientalmente perigosos pode ser inevitável (ALHASHEMl et al., 2011).

Dentre os compartimentos ecológicos abióticos, o solo é o principal estoque de elementos químicos para o ecossistema. Já a vegetação direciona a ciclagem biogeoquímica, sendo responsável pela manutenção de quantidades apreciáveis de elementos químicos nos compartimentos biológicos (MARKERT, 1991). Devido à biodiversidade inerente às florestas tropicais, o fenômeno de acumulação é complexo. Assim, num mesmo ecossistema tropical, diversas espécies podem acumular vários elementos-traço (FRANÇA et al., 2010).

0 objetivo desse trabalho foi avaliar a acumulação dos elementos químicos traços $\mathrm{Cd}$, Sb, Th e $\mathrm{U}$ na vegetação do fragmento de Mata Atlântica Refúgio Ecológico Charles Darwin - RECD em diferentes estações do ano. Para isso também foi estudado o solo sob projeção das copas das árvores.

\section{MATERIAL E MÉTODOS}

Área de estudo

O Refúgio Ecológico Charles Darwin é um remanescente de Mata Atlântica de aproximadamente 60 ha. A vegetação é Floresta Ombrófila Aberta (HIROTA et al., 2015), situada dentro da subzona de Mata Úmida de Pernambuco. A área é cortada pelo Rio Jacoca, também chamado de Tabatinga, que é perene e faz parte da Bacia Hidrográfica do Rio Botafogo.

Levantamento florístico

O levantamento florístico foi empregado como principal ferramenta para o conhecimento da vegetação do Refúgio Ecológico Charles Darwin. Para isso, foram realizadas duas expedições à área de estudo, a primeira em janeiro de 2014 e a segunda em setembro de 2014 (período chuvoso) com o objetivo de fazer um levantamento e observação da comunidade vegetal no campo e a posterior amostragem de folhas (Tabela 1). Foram observadas fisionomias diferenciadas entre duas áreas da reserva, isto é, a área alta da reserva apresentou grande quantidade de indivíduos da Família Myrtaceae, enquanto na parte baixa, observou-se um domínio de uma espécie invasora, a Pachira aquática, mais conhecida comocarolina.

Tabela 1. Listagem das espécies coletadas no Refúgio Ecológico Charles Darwin no

\begin{tabular}{|c|c|c|c|}
\hline Família & Espécie & Abreviação & $\mathrm{N}$ \\
\hline Anacardiaceae & Tapirira guianensis Aubl. & Tgui & 3 \\
\hline Araliaceae & $\begin{array}{l}\text { Schefflera morototoni(Aubl.) } \\
\text { Maguire, Steyerm. \& Frodin }\end{array}$ & Smor & 4 \\
\hline Malvaceae & Pachira aquatica Aubl. & Paqua & 11 \\
\hline Myrtaceae & Myrcia sp. & Myrt & 16 \\
\hline Simaroubaceae & Simarouba amara Aubl. & Sama & 2 \\
\hline Cyatheaceae & Cyathea microdonta(Desv.) Domin & Cyathea & 8 \\
\hline
\end{tabular}

\section{Amostragem}

Os indivíduos vegetais foram marcados por plaquetas numeradas para facilitar a localização dos indivíduos. Folhas de espécies arbóreas de maior ocorrência na região foram coletadas a partir do protocolo modificado de Ernst (1995), assim como o solo sob a projeção das copas das árvores. As coletas de material vegetal foram realizadas em janeiro e setembro de 2014. Cerca de $500 \mathrm{~g}$ de folhas das árvores com perímetro a altura do peito (PAP) acima de $15 \mathrm{~cm}$ foram retiradas com auxílio de tesoura de alto-poda. Para solo, $500 \mathrm{~g}$ de material foram amostrados sob a projeção das copas das árvores. Informações sobre herbivoria e infestação por fungos foram registradas durante as coletas. Tipicamente, as folhas apresentaram $15 \%$ de herbivoria e infestação.

Tratamento químico das amostras

As amostras de folhas foram lavadas com água de torneira, secas em estufa à $60^{\circ} \mathrm{C}$ até peso constante e moídas em moinho de rotor de titânio para a redução do tamanho das partículas $(<1 \mathrm{~mm})$. Porções analíticas com aproximadamente $1 \mathrm{~g}$ do material foram submetidas ao tratamento químico para possibilitar a análise por ICP-MS. 0 branco analítico foi analisado juntamente com as amostras, assim 
como os materiais de referência certificados. Os tratamentos químicos das amostras vegetais e geológicas utilizaram mistura de ácidos apropriada, conforme a metodologia descrita por Silva Neto (2015).

Alíquotas de $20 \mu \mathrm{l}$ das soluções de materiais de referência, amostras e brancos analíticos foram analisadas no equipamento NexION 300 ICP-MS, PerkinElmerSCIEX para a determinação de Cd, Sb, Th e U.

Fator de transferência solo-planta

Para o estudo de acumulação de elementos químicos traços na vegetação, foi considerado o fator de transferência (FT) de elementos químicos traços no sistema solo-planta:

$$
F T=\frac{C_{1}}{C_{2}}
$$

na qual,

$\mathrm{C}_{1}=$ concentração nas folhas avaliadas $\left(\mathrm{mg} \mathrm{kg}^{-1}\right)$,

$\mathrm{C}_{2}=$ concentração no substrato $\left(\mathrm{mg} \mathrm{kg}^{-1}\right)$.

Qualidade do procedimento analítico

A garantia de qualidade do procedimento analítico foi demonstrada a partir dos resultados dos materiais de referência analisados. 0 Número $E_{n}$ (Equação 2) foi empregado para a avaliação da qualidade dos procedimentos analíticos conforme a ISO 13528 (2005), em que valores entre -1 e 1 são indicativos de controle de qualidade do procedimento analítico em nível de $95 \%$ de confiança.

$$
E_{n}=\frac{V_{o b t}-V_{r e f}}{\sqrt{U_{o b t^{2}+U_{r e f}}^{2}}}
$$

na qual,

$\mathrm{V}_{\mathrm{obt}}=\mathrm{valor}$ obtido do elemento químico;

$V_{\text {ref }}=v a l o r$ de referência constante do certificado de análise dos materiais de referência certificados;

$\cup_{\text {obt }}=$ incerteza expandida em nível de $95 \%$ de confiança do valor obtido;

$U_{\text {ref }}=$ incerteza expandida em nível de $95 \%$ de confiança do valor de referência.

\section{RESULTADOS E DISCUSSÃO}

Os resultados da análise dos materiais de referência, IAEA-V-10 Hay Powder e SRM 1547 - Peach Leaves,e os materiais de referência certificados SRM 2709 - San Joaquin Soil e SRM 2711 - Montana Soil, por ICP-MS foram adequados, pois os números $E_{n}$ ficaram entre -1 e 1.Assim, demonstrou-se a qualidade do procedimento analítico em nível de $95 \%$ de confiança.

Os valores para os elementos químicos traços $\mathrm{Cd}$, Sb, Th e U no solo sob a projeção das copas das árvores estão apresentados na Tabela 2.

Tabela 2. Concentrações $\left(\mathrm{mg} \mathrm{kg}^{-1}\right)$ de elementos químicos traços determinados nas amostras de solo do RECD.

\begin{tabular}{ccccc}
\hline & $\mathrm{Cd}$ & $\mathrm{Sb}$ & $\mathrm{Th}$ & $\mathrm{U}$ \\
\hline Média & 0,06 & 0,59 & 11,0 & 23,0 \\
Máximo & 0,13 & 2,80 & 16,0 & 34,0 \\
CV\% & 38 & 92 & 27 & 27 \\
\hline
\end{tabular}

A concentração de Sb nas folhas das espécies foi bastante variável, verificando-se concentração máxima de $11 \mathrm{\mu g} \mathrm{kg}^{-1}$ em uma Schefflera morototoni, enquanto a faixa esperada em plantas terrestres é 0,1-200 $\mu \mathrm{kg}^{-1}$ (SCHÜÜRMANN; MARKERT, 1998). Mesmos os maiores valores de $\mathrm{Cd}$ determinados nas folhas estiveram também na faixa de $0,03 \mathrm{mg} \mathrm{kg}^{-1} \mathrm{a} \quad 0,5 \mathrm{mg} \mathrm{kg}^{-1}$ proposta por Schüürmann e Markert (1998). As variações de concentrações de Cd, em $\mathrm{mg} \mathrm{kg}^{-1}$ nas folhas das espécies arbóreas do RECD coletadas em janeiro e setembro de 2014 são apresentadas na Figura 1. A Cyathea microdonta no período seco apresentou valor médio de $\mathrm{Cd}$ na ordem de $0,09 \mathrm{mg} \mathrm{kg}^{-1}$, sendo esse valor de acordo com a faixa de 0,03 $0,5 \mathrm{mg} \mathrm{kg}^{-1}$ para plantas (SCHUURMANN; MARKET, 1998).

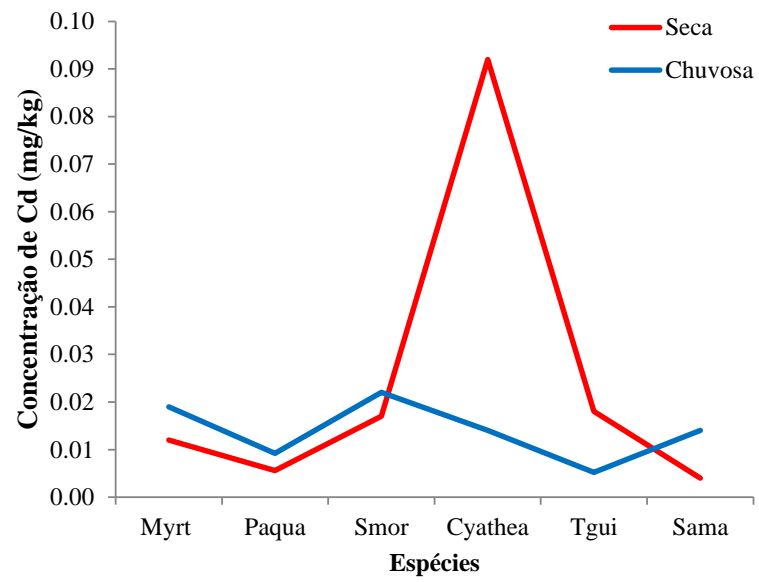

Figura 1. Concentração $\left(\mathrm{mg} \mathrm{kg}^{-1}\right)$ de $\mathrm{Cd}$, determinada nas folhas das espécies arbóreas coletadas em janeiro e setembro de 2014.

A concentração máxima de Th encontrada nas espécies analisadas no RECD foi $0,19 \mathrm{mg} \mathrm{kg}^{-1} \mathrm{em}$ Cyathea microdonta coletada no período seco (Figura 2). Os valores de concentração de Th estipulados por Schuurmann e Market (1998) variam de $0,03 \mathrm{mg} \mathrm{kg}^{-1} \mathrm{a} 1,3 \mathrm{mg} \mathrm{kg}^{-1}$. Tório pode estar associado à contaminação da superfície de folhas, assim como U, conforme o estudo realizado por Ferrari et al. (2006).

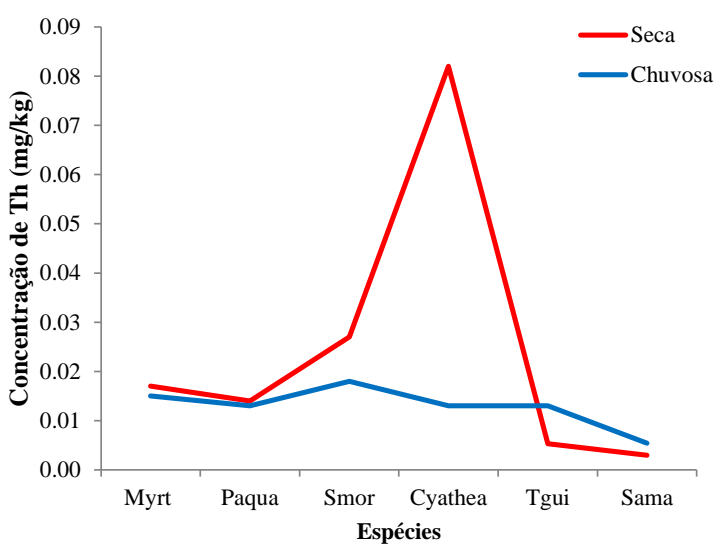

Figura 2. Concentração $\left(\mathrm{mg} \mathrm{kg}^{-1}\right)$ de Th, determinada nas folhas das espécies arbóreas coletadas em janeiro e setembro de 2014

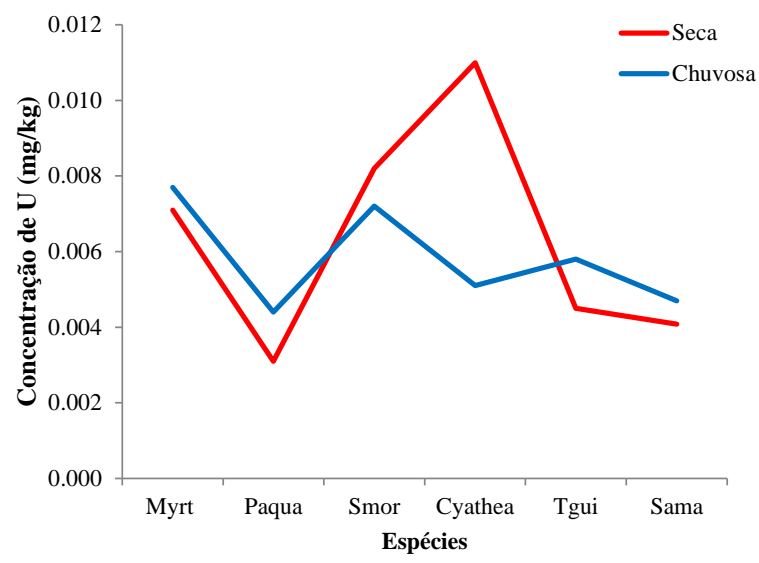

Figura 3. Concentração $\left(\mathrm{mg} \mathrm{kg}^{-1}\right)$ de $\mathrm{U}$, determinada nas folhas das espécies arbóreas coletadas em janeiro e setembro de 2014 . 
O Urânio apresentou concentração máxima nas espécies de Cyathea microdonta coletadas no período seco, $0,011 \mathrm{mg} \mathrm{kg}^{-1}$ (Figura 3), assim como observado para o elemento químico Th.

As razões entre as concentrações totais determinadas no solo sob a projeção das copas das árvores e aquelas encontradas nas folhas foram utilizadas para estimar os fatores de transferência entre os compartimentos solo e folha - FT.

A transferência de Sb ocorreu principalmente para Schefflera morototoni, em ambas estações. A transferência de Cd no sistema biogeoquímico é considerável, como representado na Figura 4. Como $\mathrm{Cd}$ não é um elemento químico essencial, é possível dizer que algumas espécies nativas não estejam conseguindo evitar a absorção deste elemento químico no solo. Contudo, a acumulação de Cd ocorreu para Simarouba amara e Myrcia sp. Futuros estudos de biomonitoração desse elemento químico poderão ser realizados utilizando, principalmente, a espécie $S$. amara. Vale ressaltar que valores de FT para este elemento químico são raros na literatura. Th e U não apresentam funções biológicas para os organismos. Mesmo assim, algumas espécies nativas apresentaram acumulação dos elementos químicos nas folhas como Cyathea microdonta, como notado por Araújo et al. (2012). Algumas samambaias são acumuladoras de radionuclídeos naturais conforme discutido por Koyama et al. (1987).
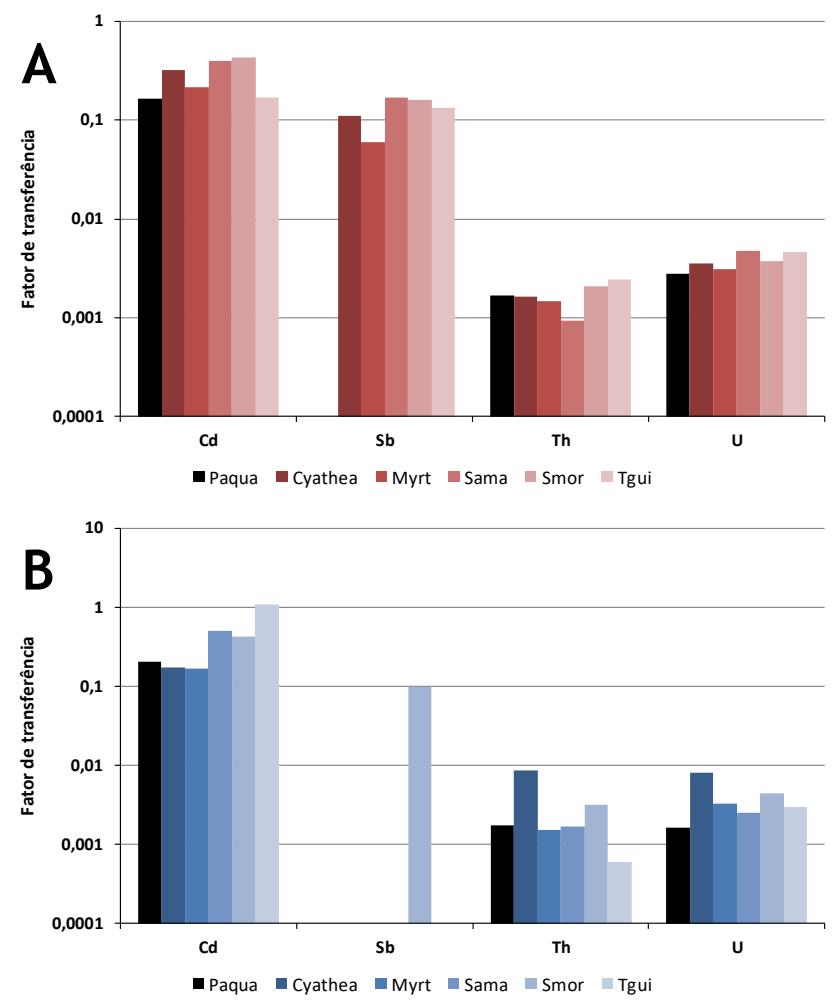

Figura 4. Fatores de transferência para as espécies arbóreas amostradas emSetembro de 2014 (A), Janeiro de 2014 (B). Paqua = Pachira aquatica, Cyathea = Cyathea macrodonta, Myrt = Myrcia sp., Sama = Simarouba amara, Smor = Schefflera morototoni, Tgui = Tapirira guianensis.

\section{CONCLUSÕES}

Este trabalho foi realizado a partir da aplicação da técnica analítica de ICP-MS. Verificou-se um possível efeito de atividades humanas sobre a acumulação de $\mathrm{Cd}$ nas folhas, ocasionando ciclagem pronunciada do elemento no Refúgio Ecológico Charles Darwin. A partir dos resultados das determinações de Th e $U$ nas espécies arbóreas nativas do fragmento de Mata Atlântica, constatou-se que os vegetais estudados apresentam capacidade de acumulação desses elementos químicos em suas folhas.

\section{REFERÊNCIAS}

ALHASHEMI, A. S. H.; KARBASSI, A. R.; KIABI, B. H.; MONAVARI, S. M.; NABAVI, S. M. B.; SEKHAVATJOU, M. S. Bioaccumulation of trace elements in trophic levels of wetland plants and waterfowl birds. Biological Trace Element Research, v.142, p.500-516, 2011.

ARAÚJO, A. L. L.; FERNANDES, E. A. N.; BACCHI, M. A.; FRANÇA, E. J. Bioaccumulation pattern of lanthanides in pteridophytes and magnoliophytes species from Atlantic Forest. Journal of Radioanalytical and Nuclear Chemistry, v.291, p.187-192, 2012.

FRANÇA, E. J. A biomonitoração da Mata Atlântica na conservação da biodiversidade: espécies arbóreas nativas acumuladoras de elementos químicos. Tese (Doutorado), Piracicaba, Centro de Energia Nuclear na Agricultura, Universidade de São Paulo, 2006, 376p.

FRANÇA, E.J.; DE NADAI FERNANDES, E.A. ; BACCHI, M.A. ; RODRIGUES, R.R. ; BODE, P. ; SAIKI, M. . Native plant bioaccumulation strategies for biomonitoring the Atlantic Forest. International Journal of Environment and Health, v. 4, p. 181-200, 2010

FAIRBROTHER, A; WENSTEL, R.; SAPPINGTON, S.; WOOD, W. Framework for metals risk assessment. Ecotoxicology Environmental Safety v.68, p.145-227, 2007.

FERRARI, A.A.; FRANÇA, E.J.; DE NADAI FERNANDES, E.A.; BACCHI, M.A. Surface contamination effects on leaf chemical composition in the Atlantic Forest. Journal of Radioanalytical and Nuclear Chemistry, v. 270, n. 1, pp. 69-73, 2006

ERNST, W.H.O. Sampling of plant material for chemical analysis. The Science of the Total Environment, v. 176, p.15-24, 1995.

HIROTA M. Atlas dos remanescentes florestais da Mata Atlântica período 2013-2014, Relatório Técnico. Fundação SOS Mata Atlântica, Instituto de Pesquisas Espaciais. São Paulo, 2015, 60p. INTERNATIONAL ORGANIZATION FOR STANDARTIZATION - ISO. ISO13528 Statistical methods for use in proficiency testing by interlaboratory comparisons. Genebra: ISO, 2005. 66p.

LUOMA, S.N.; RAINBOW, P.S. Why is metal bioaccumulation so variable? Biodynamics as a unifying concept. Environmental Science \& Technology, v.39, n.7, p.1921-1931, 2005.

MARKERT, B. Establishing of 'Reference Plant' for inorganic characterization of different plant species by chemical fingerprint. Water, Air, \& Soil Pollution, v.64, n. 3-4, p.533-538, 1991. SCHÜÜRMANN, G., MARKERT, B. Ecotoxicology. Amsterdam: John Wiley, $1998.900 \mathrm{p}$.

XU, J.; YANG, L.; WANG, Z.; DONG, G.; HUANG, J.; WANG, Y. Toxicity of copper on rice growth and accumulation of copper in rice grain in copper-contaminated soil. Chemosphere, v.62, p.602-607, 2006. 